\title{
Unmoderated Posters: Transplantation and Vascular Surgery
}

\author{
UP-55 \\ The First Report of Alemtuzumab as Rescue Therapy in Kidney- \\ pancreas Allograft Rejection \\ Rowe, Neal'; Archambault, Jason'; Aboalsamh, Ghaleb'; Maclean, Kelly²; \\ Sener, Alp'; Luke, Patrick' \\ ${ }^{1}$ Western University, London, ON, Canada; ${ }^{2}$ London Health Sciences \\ Centre, London, ON, Canada \\ Introduction: Alemtuzumab is a humanized anti-CD52 monoclonal anti- \\ body that depletes $\mathrm{T}$ and $\mathrm{B}$ lymphocytes. While the use of this agent has \\ been well established for induction immunosuppression at many centres, \\ its utilization for the treatment of kidney allograft rejection has only been \\ reported in a limited number of small series. To our knowledge, this is the \\ first report of alemtuzumab rescue therapy for mixed cellular and antibody \\ mediated rejection (AMR) in simultaneous pancreas-kidney (SPK) recipients. \\ Methods: Three SPK patients have been treated with alemtuzumab for \\ refractory mixed cellular and AMR at our centre. All patients had received \\ induction immunosuppresion with anti-thymocyte globulin. Two patients \\ were maintained on tacrolimus, mycophenolate and corticosteroid while
}

a third patient was on a steroid sparing regimen. All patients developed mixed cellular and antibody mediated rejection at various time points post-transplant. Treatment included administration of corticosteroids and anti-thymocyte globulin in conjunction with plasma exchange, intravenous immunoglobulin (IVIG), and cyclophosphamide. Despite aggressive immunosuppression, patients continued to have minimal improvement on kidney biopsy and continued to have ongoing functional decline. Each patient received alemtuzumab via peripheral line.

Results: Kidney and pancreas function stabilized (as measured by serum creatinine, lipase and amylase) with no evidence of rejection on follow-up biopsy. Our first patient, treated late, subsequently lost the allografts for poor residual function but demonstrated resolution of rejection on biopsy. Conclusions: Refractory mixed cellular and antibody mediated rejection in SPK recipients can be successfully treated with alemtuzumab. Our initial experience suggests early initiation of therapy is required for allograft salvage. However, long-term follow-up and further study is required before adopting this regimen for general use. 